\title{
25 Research Soure \\ Salvage Surgeries for Splanchnic Artery Aneurysms after Failed Endovascular Therapies: Cases Series
}

\section{Yi-Chun Lin}

China Medical University

Tzu-Chi Liao

China Medical University

\section{Long-Bin Jeng}

China Medical University Hospital

Horng-Ren Yang

China Medical University Hospital

\section{Chung-Ho Hsu}

China Medical University Hospital

Wei-Ching Lin

China Medical University Hospital

\section{Ching-Feng Wu}

China Medical University Hospital

Chun-Chieh Yeh ( $\square$ transplantyeh@gmail.com )

China Medical University Hospital

\section{Research Article}

Keywords: salvage surgery, failed endovascular therapy, giant aneurysm, mass effect, jaundice, surgery

Posted Date: February 18th, 2022

DOI: https://doi.org/10.21203/rs.3.rs-1303488/v1

License: (c) (i) This work is licensed under a Creative Commons Attribution 4.0 International License.

Read Full License 


\section{Abstract}

\section{Introduction}

Endovascular therapies are the first-line treatment for splanchnic aneurysms. However, after failed endovascular therapies or giant aneurysms with mass effects, appropriate management for splanchnic aneurysms remained inconclusive.

\section{Method}

A retrospective review was performed for consecutive patients (from 2019 to 2021) who underwent salvage surgeries following failed endovascular therapies for splanchnic artery aneurysms. Salvage operations included total aneurysmectomy with end-to-end vascular reconstruction and partial aneurysmectomy with directly closing bleeders from the intraluminal space of the aneurysms.

\section{Result}

Four patients received salvage surgeries for three false aneurysms of the celiac or superior mesenteric arteries and a true aneurysm of the common hepatic artery. The causes of failed endovascular therapies included coil migration, insufficient space for safely deploying the endovascular stent or persistent mass effect from the thrombosed giant aneurysm. Mean hospital stay was five days (range, 3-7 days), with 0\% of morbidity and mortality rate and $100 \%$ of symptoms improvement. During the follow-up period (mean, 8.75 months; range, 4-15 months), one patient suffered a small residual asymptomatic celiac artery aneurysm (8 $\mathrm{mm}$ in diameter) and was treated conservatively due to underlying liver cirrhosis.

\section{Conclusion}

Surgical management is a feasible, effective, and safe alternative for splanchnic aneurysms undergoing failed endovascular therapies.

\section{Introduction}

Splanchnic arterial aneurysms are rare but can be a potentially lethal disease. The incidence of splanchnic arterial aneurysms is estimated to be $0.1-2 \%(1,2)$. Approximately $25 \%$ of splanchnic arterial aneurysms initially presented as rupture, and the mortality rate after rupture is more than $10 \%$, highlighting the importance of timely diagnosis and adequate management (2-5). Splanchnic aneurysms include true aneurysms with all three layers of the vascular wall and false aneurysms lined by adventitia or perivascular tissue (3). True and false splanchnic aneurysms differ in pathophysiology (i.e., arteriosclerosis/ autoimmune disease/ collagen vascular disease vs. trauma/ infection/ inflammation), and risk of spontaneous rupture (i.e., occurring mostly while diameter $>2 \mathrm{~cm}$ vs. occurring regardless of size) $(2,3,6)$. However, gastroduodenal artery (GDA), superior mesenteric artery (SMA), and pancreaticoduodenal artery (PDA) true aneurysms differ from other splanchnic true aneurysms (e.g., splenic arterial aneurysm) by a higher risk of ruptured aneurysm regardless of its size (5). Similarly, a 
false splanchnic aneurysm has a significant risk of spontaneous rupture irrespective of its size $(5,7)$. Thus, selected true splanchnic aneurysms (e.g., any GDA, SMA, PDA aneurysms, aneurysm $>2 \mathrm{~cm}$, or symptomatic aneurysms) and false aneurysms may warrant timely and appropriate intervention to avoid aneurysm rupture $(2,3,5,6)$.

Previous studies reported that their strategies for treating splanchnic aneurysms varied depending on the anatomical location, size, pathophysiological processes, collateral flow, true or false aneurysm, ruptured or intact aneurysms, and hemodynamic status $(2,3,5,6,8)$. Because of minimally invasive characteristics, percutaneous endovascular therapy has become the first treatment modality for splanchnic aneurysms; however, around $10 \%$ of cases underwent failed endovascular therapy due to incomplete exclusion of aneurysm or difficulty in catheterization (6). Tortuous parent vessels, irregularity of the aneurysm, and inadequate proximal or distal landing zone also predispose failed endovascular therapy in splanchnic aneurysms (2). Surgical intervention is the previously definitive and currently alternative treatment for splanchnic aneurysms with higher morbidity, comparable mortality but less reintervention rate than endovascular therapies $(1,9,10)$. However, most previous studies did not comprehensively describe surgical details (i.e., when and how to choose either aneurysmectomy with endto-end vascular reconstruction or directly repairing defect of the vascular wall) or the prognosis of mass effect caused by a thrombosed large aneurysm.

The unique anatomic position of the splanchnic aneurysm makes us have to take special consideration for its management. For example, a larger-sized splanchnic aneurysm may cause compression effects such as pancreatitis, obstructive jaundice, or intestinal obstruction (11-13). Nonetheless, mass effects caused by aneurysms were less discussed before. In addition, tortuous vascular anatomy makes endovascular therapy for splanchnic aneurysms challenging and unexpectedly risky (e.g., non-target organ ischemia). Most studies concerning splanchnic aneurysms were published from the perspectives of interventional radiologists, cardiovascular physicians, or surgeons. Hence, from the general surgeon's perspective, we would like to share our successful experience managing splanchnic aneurysms, particularly for giant aneurysms and aneurysms undergoing failed endovascular therapy.

Herein, we present four splanchnic aneurysms (i.e., celiac artery aneurysm, common hepatic artery aneurysm, superior mesenteric artery branch aneurysm, and dorsal pancreatic artery aneurysm) with different sizes, locations, etiologies, symptoms, and corresponding treatments. We also proposed a decision-making flowchart (Figure 5) according to the pathophysiology (i.e., true or false) and mass effect to achieve a tailored integrated therapy for the splanchnic aneurysms.

\section{Methods}

Between 2019 and 2021, we consecutively enrolled patients who received surgical salvages after failed endovascular therapies for splanchnic arterial aneurysms in a tertiary university hospital (China Medical University Hospital, Taichung, Taiwan). The splanchnic aneurysms were mainly diagnosed by computed 
tomography (CT). Corresponding CT reports included its location, size, neck length, signs of rupture, and the existence of feeding arteries.

Perioperative data and interventions were collected after retrospectively reviewing medical records. Preoperative data contained symptoms, comorbidity, risk factors, and anatomical characteristics of the aneurysms and the causes of failed endovascular therapies; perioperative variables included surgical details for vascular repair or reconstruction; post-operative data included the surgery-related complications and clinical outcomes of the aneurysms. The ethical issues of this study had been reviewed and approved by the institutional review board (IRB) at China Medical University Hospital (IRB no.: CMUH110-REC1-182).

\section{Salvage Surgical Technique}

If the aneurysm is located outside the parenchyma of solid organs, we will try to control the aneurysm's proximal and distal vascular end first, followed by aneurysmectomy and end-to-end anastomosis. Nevertheless, when encountering a hostile abdomen (e.g., false aneurysms secondary to chronic pancreatitis), we perform partial aneurysmectomy and close the orifice of feeding vessels from the intraluminal space of the aneurysm. After the surgical and anesthetic team are well prepared for bleeding control and rapid blood transfusion, we incise the aneurysm, compress the major bleeder by the fingertips, and immediately seal the orifice from the intraluminal space with polypropylene suturing. In the saccular aneurysm, we would suture carefully to avoid suturing the opposite vascular wall so that the patency of the lumen can still be maintained. In the fusiform aneurysm, we can seal the two bleeders from intraluminal space after opening the aneurysm without compromising organ perfusion due to abundant collateral circulation in the splanchnic vascular system. However, in some nonexpendable vessels (e.g., SMA trunk, proper hepatic artery), aneurysmectomy with the restoration of blood flow by vascular reconstruction is still needed. The thrombosed hematoma will be evacuated, and an omentum patch will pack the dead space within the aneurysm to avoid infected fluid accumulation. If the aneurysm is embedded within the pancreas region, the edge of the aneurysm wall will be secured by running suturing to avoid pancreas juice leakage.

\section{Results}

Out of four patients, two males and two females mean 61.3 years (47-74 years). Three out of four lesions were false aneurysms of the celiac artery, superior mesenteric artery, and dorsal pancreatic artery, respectively. One case was a true aneurysm of a common hepatic artery involving trifurcation of the gastroduodenal artery, proper hepatic artery, and common hepatic artery. The mean diameter of the aneurysms was $77.5 \mathrm{~mm}$ (range, $30-160 \mathrm{~mm}$ ). Three patients $(75 \%)$ presented with abdominal fullness, or along with jaundice or abdominal pain additionally. In the asymptomatic patient, the lesion was detected by sonography incidentally while surveying Lipitor-related hepatitis. (Table 1) 
Table 1

Patients' characteristics.

\begin{tabular}{|c|c|c|c|c|}
\hline Case & 1 & 2 & 3 & 4 \\
\hline Age & 47 & 59 & 65 & 74 \\
\hline Gender & Male & Female & Male & Female \\
\hline $\begin{array}{l}\text { TRUE } \\
\text { Or } \\
\text { FALSE } \\
\text { aneurysm }\end{array}$ & FALSE & TRUE & FALSE & FALSE \\
\hline Etiology & $\begin{array}{l}\text { Chronic } \\
\text { pancreatitis }\end{array}$ & Undetermined & Chronic pancreatitis & Undetermined \\
\hline $\begin{array}{l}\text { Initial } \\
\text { presentation }\end{array}$ & $\begin{array}{l}\text { Abdominal } \\
\text { fullness }\end{array}$ & Asymptomatic & $\begin{array}{l}\text { Jaundice } \\
\& \\
\text { Abdominal fullness }\end{array}$ & $\begin{array}{l}\text { Abdominal } \\
\text { pain \& fullness }\end{array}$ \\
\hline $\begin{array}{l}\text { Location } \\
\text { (artery) }\end{array}$ & Celiac & Common hepatic & Superior mesenteric & $\begin{array}{l}\text { Dorsal } \\
\text { pancreatic }\end{array}$ \\
\hline $\begin{array}{l}\text { Size } \\
(\mathrm{mm})\end{array}$ & $60 * 60$ & $30 * 30$ & $60 * 60$ & $160 * 140$ \\
\hline $\begin{array}{l}\text { Causes of } \\
\text { failed } \\
\text { endovascular } \\
\text { therapy }\end{array}$ & $\begin{array}{l}\text { - Tortuous } \\
\text { vessels } \\
\text { - Insufficient } \\
\text { landing zone }\end{array}$ & $\begin{array}{l}\text { - Tortuous } \\
\text { vessels } \\
\text { - Risk of } \\
\text { occluding } \\
\text { the right hepatic } \\
\text { artery after } \\
\text { deploying stent }\end{array}$ & $\begin{array}{l}\text { - Coils migration } \\
\text { - Risk of losing } \\
\text { nearby SMA } \\
\text { branches after } \\
\text { deploying stent }\end{array}$ & $\begin{array}{l}\text { - Coils migration } \\
\text {-Persistent mass } \\
\text { effect }\end{array}$ \\
\hline $\begin{array}{l}\text { Surgical } \\
\text { therapy }\end{array}$ & $\begin{array}{l}\text { Partial } \\
\text { aneurysmectomy } \\
\text { with direct repair }\end{array}$ & $\begin{array}{l}\text { Aneurysmectomy } \\
\text { with end-to-end } \\
\text { anastomosis }\end{array}$ & $\begin{array}{l}\text { Partial } \\
\text { aneurysmectomy } \\
\text { with direct repair }\end{array}$ & $\begin{array}{l}\text { Partial } \\
\text { aneurysmectomy } \\
\text { with direct repair }\end{array}$ \\
\hline Outcome & $\begin{array}{l}\text { Partial resolution } \\
\text { with } \\
\text { residual false } \\
\text { aneurysm } \\
8 * 8 \mathrm{~mm}\end{array}$ & $\begin{array}{l}\text { Complete } \\
\text { resolution }\end{array}$ & Complete resolution & $\begin{array}{l}\text { Complete } \\
\text { resolution }\end{array}$ \\
\hline $\begin{array}{l}\text { Follow-up } \\
\text { (months) }\end{array}$ & 12 & 15 & 4 & 4 \\
\hline
\end{tabular}


After failed endovascular therapies, all four cases underwent salvage operations to treat their true/false aneurysms of different locations. Two patients received coil embolization but failed due to coil migration. The other two patients failed endovascular trials due to the tortuous vascular anatomy, an insufficient landing zone, and an increased risk of occluding the nearby arteries if deploying the stent.

As for the types of open surgery, only one patient underwent complete aneurysmectomy with end-to-end anastomosis for the true aneurysm at the trifurcation of common hepatic artery/ proper hepatic artery/ gastroduodenal artery. The other three patients of false aneurysms underwent partial aneurysmectomy with a direct repair. The average hospital stay length was five days (3-7 days), with $0 \%$ of surgery-related morbidity and mortality.

In a median follow-up of 8.75 months (range, 4-15 months), 75\% (3/4) of patients did not have recurrent aneurysms, but only one case has partial resolution with asymptomatic remaining false aneurysm (8mm in diameter). He was treated conservatively due to the severe underlying comorbidity (alcoholic liver cirrhosis, child B), and the patient's initial abdominal fullness indeed improved after the surgical salvage. The re-intervention rate was $0 \%$.

\section{Case Presentation}

\section{Case1- Celiac artery false aneurysm}

A 47-year-old man with underlying alcoholism-related liver cirrhosis and chronic pancreatitis presented abdominal fullness for one week. The biochemical tests showed mildly increased liver enzyme (aspartate aminotransferase $102 \mathrm{U} / \mathrm{L}$, total bilirubin $28.91 \mu \mathrm{mol} / \mathrm{L}$, direct bilirubin $16.08 \mu \mathrm{mol} / \mathrm{L}$ ). Abdominal CT demonstrated a celiac artery false aneurysm. (Figure 1A). Angiography showed a false aneurysm $6 \mathrm{~cm}$ in diameter arising from the bifurcation of the celiac artery. (Figure 1B).

He underwent a failed endovascular trial because of tortuous vascular anatomy and insufficient landing zone for stent deployment. Then, we offered salvage laparotomy for partial aneurysmectomy and directly repairing the neck of the false aneurysm (Figure 1C,1D). After six months, abdominal CT showed a remaining small false aneurysm around $8 \mathrm{~mm}$ in diameter (Figure 1E). Considering underlying cirrhosis with liver decompensation, he chose conservative treatment by well-controlling blood pressure, and the aneurysm did not change its size anymore for the post-operative nine months.

\section{Case2- Common hepatic artery true aneurysm}

A 59-year-old woman with underlying type I Diabetes Mellitus and dyslipidemia was accidentally diagnosed with a pancreatic head mass lesion by abdominal sonography while surveying Lipitor- related hepatitis. Endoscopic ultrasonography showed an aneurysm $3 \mathrm{~cm}$ in diameter connecting with the common hepatic artery (Figure 2A). The CT scan and CT angiography revealed an aneurysm located between the trifurcation of the common hepatic artery, proper hepatic artery, and GDA (Figure 2B and 2C). Endovascular therapy failed because of the tortuous vascular anatomy and the risk of occluding the right 
hepatic artery after deploying the stent. Thus, we offered open surgery to resect the aneurysm and perform an end-to-end anastomosis between the common hepatic artery and the common orifice of the proper hepatic artery and GDA (Fig. 2D, 2E). No more recurrent aneurysm was observed in a 15-month follow-up.

\section{Case3- Superior mesenteric artery false aneurysm}

A 65-year-old man with underlying alcohol-related chronic pancreatitis presented jaundice for two weeks. Laboratory data showed obstructive jaundice (total bilirubin $196.35 \mu \mathrm{mol} / \mathrm{L}$, direct bilirubin 125.71 $\mu \mathrm{mol} / \mathrm{L}$, alkaline phosphatase $454 \mathrm{IU} / \mathrm{L}, \gamma$ - Glutamyltransferase $645 \mathrm{IU} / \mathrm{L}$, lipase $2009 \mathrm{U} / \mathrm{L}$ ). Abdominal sonography revealed a peri-pancreatic aneurysm with a "ying-yang" sign (Figure 3A). Abdominal CT showed a 6-cm pseudoaneurysm at the pancreatic head compressing the distal common bile duct with a dilated extra-hepatic biliary tract and a distended gallbladder (Figure 3B).

Coil angioembolization failed due to coils migration (Figure 3C). An endovascular stent cannot be applied due to the risk of losing the nearby SMA branches. Thus, we offered a salvage operation by ligating the proximal feeding vessel from SMA, partial aneurysmectomy, and sealing the orifice of the distal feeding vessels from the intraluminal space of the aneurysm (Figure 3D). The stenosis of the common bile duct resolved smoothly after excising the aneurysm and placing a temporal biliary tree internal stent for four months. No more recurrent aneurysm was noticed at follow-up CT on the fourth post-operative month.

\section{Case4- Dorsal pancreatic artery false aneurysm}

A 74-year-old woman without an underlying disease presented periumbilical pain for two months. Within two months, she had reduced appetites, easy satiety, and a bodyweight loss of $4 \mathrm{Kg}$. Abdominal CT showed a peripancreatic false aneurysm $16 \mathrm{~cm}$ in diameter with intraluminal partial thrombosis that caused external compression to stomach and duodenum(Figure 4A, 4B). Angiography confirmed the aneurysm arising from the dorsal pancreatic artery, occluded proximally by coil angioembolization. Due to high intraluminal vascular flow, a coil migration to the distal splenic artery occurred. Considering residual mass effect and incomplete exclusion of the aneurysm, we offered a salvage operation for partial aneurysmectomy and direct ligating the orifice of feeding vessels from the intraluminal space of the aneurysm(Figure 4C). The patient got complete resolution of the compression signs and was discharged uneventfully on post-operative day 7 . No more recurrent aneurysm was observed in the postoperative four months.

Based on the literature review and our experience, we proposed an algorithm to manage splanchnic aneurysms appropriately depending on their location, size, and clinical situations (Figure 5).

\section{Discussion}

\section{Endovascular intervention}


Thanks to the minimally invasive characteristics, endovascular therapy has been the mainstream in the therapy of splanchnic aneurysms. Endovascular therapies are superior to surgical interventions in the hostile abdomen, where dense adhesion occurs due to recent surgeries or chronic inflammation. The commonly used endovascular therapies for the aneurysm include coil angioembolization to exclude aneurysms in expendable vessels and endovascular stent to keep the patency of the nonexpendable parent vessel and the exclusion of the aneurysm concomitantly (14-16). The feasibility of endovascular therapy in splanchnic aneurysms is determined by vascular anatomy $(14,16)$. Needs for a sufficient working landing zone and the risk of blocking nearby vascular branches limit the application of endovascular stents in splanchnic aneurysms (16-18).

On the other hand, the risk of coil angioembolization includes coil migration-related non-target ischemia (19); incomplete exclusion of the aneurysm (16); requirement of multiple coils for sac packing in giant aneurysms; incomplete thrombosis in ruptured aneurysms when hemorrhagic shock-related coagulopathy develops (16). Corresponding to the current guideline, our patients received endovascular trials first, followed by salvage operations if endovascular therapy failed (5). Insufficient landing zone for deploying stent due to the tortuous vascular anatomy and high risk of coil migration-related non-target ischemia due to high intra-luminal vascular flow accounted for failed endovascular therapies in cases - 1

and 2. Fear of losing nearby critical vascular branches after deploying endovascular stent and incomplete exclusion of the aneurysms by coil angioembolization accounted for failed case - 3 . In case - 4 , a residual arterial backflow and a mass effect caused by the giant hematoma after proximal angioembolization made us have to offer a salvage surgery. Therefore, we considered that the success rate of endovascular therapies in the splanchnic aneurysms near the pancreas might not be so high as other positions because of its unique anatomic positions (i.e., tortuous, redundant, highly variable, and high-flow vascular system).

\section{Surgical intervention}

While the endovascular approach fails, surgical intervention will be a choice. Conventionally, to isolate and control proximal and distal vessels, followed by aneurysmectomy and end-to-end vascular reconstruction will be a standard procedure to treat the aneurysm and maintain vascular continuity (5). Nonetheless, the procedure needs to create a proper surgical field for engaging it, and it may cause ungrateful bleeding and unexpected injuries to the surrounding organs, particularly in chronic pancreatitis, which is the most common etiology of false splanchnic aneurysms, and also accounted for $50 \%$ of our patients (8). Moreover, lengthy adhesiolysis in the hostile abdomen secondary to chronic pancreatitis or recent operations is often associated with an increased risk of perioperative complications (20-22). Hence, this might be why open surgery carries a higher complication rate than endovascular therapy in some studies $(4,10)$.

Since endovascular therapy is widespread, studies regarding surgical interventions for splanchnic aneurysms are relatively few. Pulli $\mathrm{R}$ et al. published their 25-year experience in managing splanchnic aneurysms (1). As a cardiovascular surgical team, their surgical goal focused on restoring normal 
vascular flow. Thus, around $50 \%$ of cases received aneurysmectomy with end-to-end vascular anastomosis even in the areas where abundant collateral flow exists (e.g., peripancreatic artery, splenic artery, or gastric arteries). Besides, they also performed aneurysmectomy with primary or patch closure or arterial ligature. However, they did not mention the criteria for choosing different surgical approaches. They reported that surgical intervention has good long-term outcomes. However, they also reported a case of mortality secondary to post-operative pancreatitis after they did aneurysmectomy with end-to-end anastomosis for splenic artery aneurysm. This report corresponded well to our assumption that extensive dissection in the hostile abdomen for preparing end-to-end vascular reconstruction may predispose the accidental injury and cause subsequent morbidity and mortality.

In the perspective of general surgeons, we have a slightly different philosophy to treat splanchnic aneurysms. If the aneurysm is embedded inside the parenchyma and cannot be controlled by endovascular therapy, we favor excising the aneurysm along with end-organ (e.g., splenectomy for the aneurysm inside the spleen). If the aneurysm is located outside the parenchyma of solid organs, we will try to control the aneurysm's proximal and distal vascular end first, followed by aneurysmectomy and end-to-end anastomosis (23). Nevertheless, when dissecting the vessels in the hostile abdomen is inviable, we adopted the surgical concept of directly repairing femoral and iliac artery aneurysm (24-26). Even in a dense adhesion region, directly opening the vascular wall of the aneurysm and closing the orifice of the feeding vessels like distal and proximal embolization by coils (i.e., Sandwich technique) can still be done quickly. Since the collateral circulation in the peripancreatic area is abundant, except for some nonexpendable vessels (e.g., SMA trunk, proper hepatic artery), proximal and distal ligation of the aneurysm can be done safely without compromising perfusion of the distal organ (e.g., our case - 3 and 4: SMA branch aneurysm and dorsal pancreatic aneurysm). We believe that our criteria of selecting surgical approaches for splanchnic aneurysms could avoid accidental injuries caused by surgical dissection at the hostile abdomen and reduce the risk of perioperative morbidity.

\section{Mass effect}

Either surgery or endovascular therapy as the most appropriate treatment for giant splanchnic aneurysms (i.e., diameter $>5 \mathrm{~cm}$ ) remains undetermined, particularly for the aneurysm with concomitant mass effect $(2,33-36)$. Some authors preferred open surgery because embolization with thrombosis of the aneurysm cannot resolve the mass effect immediately (33). However, successful remission of obstructive jaundice by embolization to the splanchnic aneurysms was also reported sporadically $(2,34,37)$. Tipaldi et al. displayed their experience using endovascular therapy to giant splanchnic artery aneurysms in 11 cases, and one case obtained resolution of jaundice after embolizing the aneurysm (2). However, considering technically challenging and high cost, they also stated that endovascular therapy should be listed as the first-line treatment for the giant aneurysm only when the necessary expertise and resources are fully available and the patients' hemodynamic condition is stable (2).

Back to our experience, we think the giant aneurysm does not necessarily need surgical intervention if endovascular therapy is available and successful. However, if the giant aneurysm occurs in association 
with mass effect (e.g., obstructive jaundice or bowel obstruction), surgical intervention could be an alternative to provide immediate resolution of the aneurysm and its associated compression. Following our treatment philosophy, incising and exploring the giant aneurysm, suturing the bleeder from the inner lumen, and emptying the contained hematoma for immediate resolution of mass effect would be feasible and straightforward for any well-prepared surgical team. Noteworthy, because obstructive jaundice may be caused by either the giant aneurysm or underlying chronic pancreatitis, surgeons should rule out residual biliary stricture by intraoperative cholangiography after successful treatment for the giant aneurysm with associated jaundice (33). If residual biliary stricture exists, concomitant bilioenteric bypass or post-operative endo-biliary drainage may be needed.

\section{Recurrent aneurysms}

The residual or recurrent aneurysm after the primary surgical repair has rarely been discussed. After initial surgical repair, re-operation for a residual aneurysm is not always technically feasible because of significant inflammation and adhesion after recent operations (22). Instead, endovascular therapy for the post-operative false aneurysm has been widely accepted due to its efficacy and safety (22). Sachdev et al. and Spiliopoulos et al. both highlighted a highly successful rate of the repeated endovascular procedure after incomplete exclusion of splanchnic aneurysms following initial endovascular treatment $(7,38)$. Therefore, we consider endovascular therapy the best choice after incomplete exclusion of the aneurysm following open vascular surgery. However, if the patient suffers an asymptomatic, small-sized aneurysm with significant medical comorbidities or limited life expectancy, we could keep close observation rather than reinterventions, unless the aneurysm show interval growth from the surveillance imaging (5). In our case - 1 (celiac artery aneurysm), an 8-mm residual aneurysm was found six months later. Given the small size $(<2 \mathrm{~cm})$ of the aneurysm, asymptomatic status, and patient's limited life expectancy due to severe liver cirrhosis, we adopted conservative treatment with blood pressure control and regular follow-up with serial imaging studies, which is consistent with the latest guideline of the management of splanchnic aneurysms (5).

\section{Limitation}

The limitation of this study includes a small number of cases and the lack of long-term follow-up. Nevertheless, this study focuses on the appropriate management after failed endovascular therapy for splanchnic aneurysms and giant aneurysms with associated mass effects. Because both conditions are rarely discussed, our successful experiences on salvage surgeries for splanchnic aneurysms with failed endovascular therapies and its short-term outcomes are still valuable.

\section{Summary}

In summary, selected true or all false splanchnic aneurysms warrant timely intervention. Surgical management is a feasible, effective, and safe alternative for splanchnic aneurysms undergoing failed endovascular therapy or for giant aneurysms with associated mass effects. 


\section{Declarations}

\section{Author contribution:}

Yi-Chun Lin and Tzu-Chi Liao contributed equally as co-first authors and are responsible for drafting the manuscript and data collection.

Chun-Chieh Yeh and Ching-Feng Wu contributed equally as co-corresponding authors and are responsible for study concept and design, analysis and interpretation of data, obtaining funding.

Long-Bin Jeng, Horng-Ren Yang, Chung-Ho Hsu, Wei-Ching Lin are responsible for study supervision.

\section{Competing interests}

The authors declare no competing interests.

Correspondence and requests for materials should be addressed to Chun-Chieh Yeh.

\section{Data availability}

All data generated or analysed during this study are included in this published article.

\section{Informed consent}

We have already received a formal IRB exemption before we proceed with this observational study. Informed consent was obtained from all subjects and the data was de-identified.

\section{Announcement}

We confirm that all methods of our research were carried out in accordance with relevant guidelines and regulations

\section{References}

1. Pulli R, Dorigo W, Troisi N, Pratesi G, Innocenti AA, Pratesi C. Surgical treatment of visceral artery aneurysms: a 25-year experience. J Vasc Surg. 2008;48(2):334-42.

2. Tipaldi MA, Krokidis M, Orgera G, Pignatelli M, Ronconi E, Laurino F, et al. Endovascular management of giant visceral artery aneurysms. Scientific Reports. 2021;11(1):1-6.

3. Pasha SF, Gloviczki P, Stanson AW, Kamath PS, editors. Splanchnic artery aneurysms. Mayo Clinic Proceedings; 2007: Elsevier.

4. Shukla AJ, Eid R, Fish L, Avgerinos E, Marone L, Makaroun M, et al. Contemporary outcomes of intact and ruptured visceral artery aneurysms. J Vasc Surg. 2015;61(6):1442-8.

5. Chaer RA, Abularrage CJ, Coleman DM, Eslami MH, Kashyap VS, Rockman C, et al. The Society for Vascular Surgery clinical practice guidelines on the management of visceral aneurysms. J Vasc 
Surg. 2020;72(1):3S-39S.

6. Tetreau R, Beji H, Henry L, Valette PJ, Pilleul F. Arterial splanchnic aneurysms: Presentation, treatment and outcome in 112 patients. Diagnostic and Interventional Imaging. 2016;97(1):81-90.

7. Spiliopoulos S, Sabharwal T, Karnabatidis D, Brountzos E, Katsanos K, Krokidis M, et al. Endovascular treatment of visceral aneurysms and pseudoaneurysms: long-term outcomes from a multicenter European study. Cardiovascular and interventional radiology. 2012;35(6):1315-25.

8. Pang TC, Maher R, Gananadha S, Hugh TJ, Samra JS. Peripancreatic pseudoaneurysms: a management-based classification system. Surgical endoscopy. 2014;28(7):2027-38.

9. Marone EM, Mascia D, Kahlberg A, Brioschi C, Tshomba Y, Chiesa R. Is open repair still the gold standard in visceral artery aneurysm management? Annals of vascular surgery. 2011;25(7):936-46.

10. Barrionuevo P, Malas MB, Nejim B, Haddad A, Morrow A, Ponce O, et al. A systematic review and meta-analysis of the management of visceral artery aneurysms. J Vasc Surg. 2020;72(1):40S-5S.

11. Androulakakis Z, Paspatis G, Hatzidakis A, Kokkinaki M, Papanicolaou N, Grammatikakis I, et al. Gastric outlet obstruction caused by a giant gastroduodenal artery aneurysm: a case report. European journal of gastroenterology \& hepatology. 2001;13(1):59-61.

12. Saftoiu A, lordache S, Ciurea T, Dumitrescu D, Popescu M, Stoica Z. Pancreatic pseudoaneurysm of the superior mesenteric artery complicated with obstructive jaundice. A case report. JOP. 2005;6(1):29-35.

13. Chapman BM, Bolton JS, Gioe SM, Conway WC. Gastroduodenal artery pseudoaneurysm causing obstructive jaundice. Ochsner Journal. 2021;21(1):104-7.

14. Jesinger RA, Thoreson AA, Lamba R. Abdominal and pelvic aneurysms and pseudoaneurysms: imaging review with clinical, radiologic, and treatment correlation. Radiographics. 2013;33(3):E71E96.

15. Madhusudhan KS, Venkatesh HA, Gamanagatti S, Garg P, Srivastava DN. Interventional radiology is the management of visceral artery pseudoaneurysms: a review of techniques and embolic materials. Korean journal of radiology. 2016;17(3):351.

16. Zabicki B, Limphaibool N, Holstad MJV, Juszkat R. Endovascular management of pancreatitisrelated pseudoaneurysms: A review of techniques. PLoS One. 2018;13(1):e0191998.

17. Lopera JE, editor embolization in trauma: principles and techniques. Seminars in interventional radiology; 2010: Thieme Medical Publishers.

18. Alsafi A, Bicknell CD, Rudarakanchana N, Kashef E, Gibbs RG, Cheshire NJ, et al. Endovascular treatment of thoracic aortic aneurysms with a short proximal landing zone using scalloped endografts. J Vasc Surg. 2014;60(6):1499-506.

19. Leyon JJ, Littlehales T, Rangarajan B, Hoey ET, Ganeshan A. Endovascular embolization: review of currently available embolization agents. Current problems in diagnostic radiology. 2014;43(1):35-53.

20. Zyromski NJ, Vieira C, Stecker M, Nakeeb A, Pitt HA, Lillemoe KD, et al. Improved outcomes in postoperative and pancreatitis-related visceral pseudoaneurysms. Journal of Gastrointestinal Surgery. 
2007;11(1):50-5.

21. Ten Broek RP, Issa Y, van Santbrink EJ, Bouvy ND, Kruitwagen RF, Jeekel J, et al. Burden of adhesions in abdominal and pelvic surgery: systematic review and meta-analysis. Bmj. 2013;347.

22. Biondetti P, Fumarola EM, lerardi AM, Carrafiello G. Bleeding complications after pancreatic surgery: interventional radiology management. Gland surgery. 2019;8(2):150.

23. Grego F, Lepidi S, Ragazzi R, lurilli V, Stramana R, Deriu G. Visceral artery aneurysms: a single-center experience. Cardiovascular surgery. 2003;11(1):19-25.

24. Krupski WC, Selzman CH, Floridia R, Strecker PK, Nehler MR, Whitehill TA. Contemporary management of isolated iliac aneurysms. J Vasc Surg. 1998;28(1):1-13.

25. Moini M, Rasouli M, Rayatzadeh H, Sheikholeslami G. Management of femoral artery pseudoaneurysms in Iran: a single centre report of 50 cases. Acta Chirurgica Belgica. 2008;108(2):226-30.

26. Haseen MA, Faizan M, Beg MH, Asif N, Khalid S. Femoral artery pseudoaneurysm following trivial trauma: a rare case scenario. Indian Journal of Thoracic and Cardiovascular Surgery. 2015;31(1):34-7.

27. Saltzberg SS, Maldonado TS, Lamparello PJ, Cayne NS, Nalbandian MM, Rosen RJ, et al. Is endovascular therapy the preferred treatment for all visceral artery aneurysms? Annals of Vascular Surgery. 2005;19(4):507-15.

28. Tulsyan N, Kashyap VS, Greenberg RK, Sarac TP, Clair DG, Pierce G, et al. The endovascular management of visceral artery aneurysms and pseudoaneurysms. J Vasc Surg. 2007;45(2):276-83.

29. Hemp JH, Sabri SS. Endovascular management of visceral arterial aneurysms. Techniques in vascular and interventional radiology. 2015;18(1):14-23.

30. Bouglé A, Harrois A, Duranteau J. Resuscitative strategies in traumatic hemorrhagic shock. Annals of intensive care. 2013;3(1):1-9.

31. Coccolini F, Montori G, Catena F, Kluger Y, Biffl W, Moore EE, et al. Splenic trauma: WSES classification and guidelines for adult and pediatric patients. World Journal of Emergency Surgery. 2017;12(1):1-26.

32. Subcommittee A, Group IAW. Advanced trauma life support (ATLS®): the ninth edition. The journal of trauma and acute care surgery. 2013;74(5):1363-6.

33. Kossak J, Janik J, Debski J, Rytlewski R, Salacinski A. Pseudoaneurysm of the gastroduodenal artery as a cause of obstructive jaundice. Med Sci Monit. 2001;7(4):759-61.

34. Dönmez H, Men S, Dilli A, Soylu SÖ, Hekimoğlu B. Giant gastroduodenal artery pseudoaneurysm due to polyarteritis nodosa as a cause of obstructive jaundice: imaging findings and coil embolization results. Cardiovascular and interventional radiology. 2005;28(6):850-3.

35. Akbulut S, Otan E. Management of giant splenic artery aneurysm: comprehensive literature review. Medicine. 2015;94(27). 
36. Hamid HK, Abd Elaziz AS, Spiliopoulos S, Zabicki B, Tetreau R, Piffaretti G, et al. Giant Splenic Artery Pseudoaneurysms: Two Case Reports and Cumulative Review of the Literature. Annals of vascular surgery. 2020;64:382-8.

37. Blomley M, Jackson J. Case report: a gastroduodenal artery pseudoaneurysm presenting with obstructive jaundice and treated by arterial embolization. Clinical radiology. 1994;49(10):715-8.

38. Sachdev U, Baril DT, Ellozy SH, Lookstein RA, Silverberg D, Jacobs TS, et al. Management of aneurysms involving branches of the celiac and superior mesenteric arteries: a comparison of surgical and endovascular therapy. J Vasc Surg. 2006;44(4):718-24.

\section{Figures}

(a)
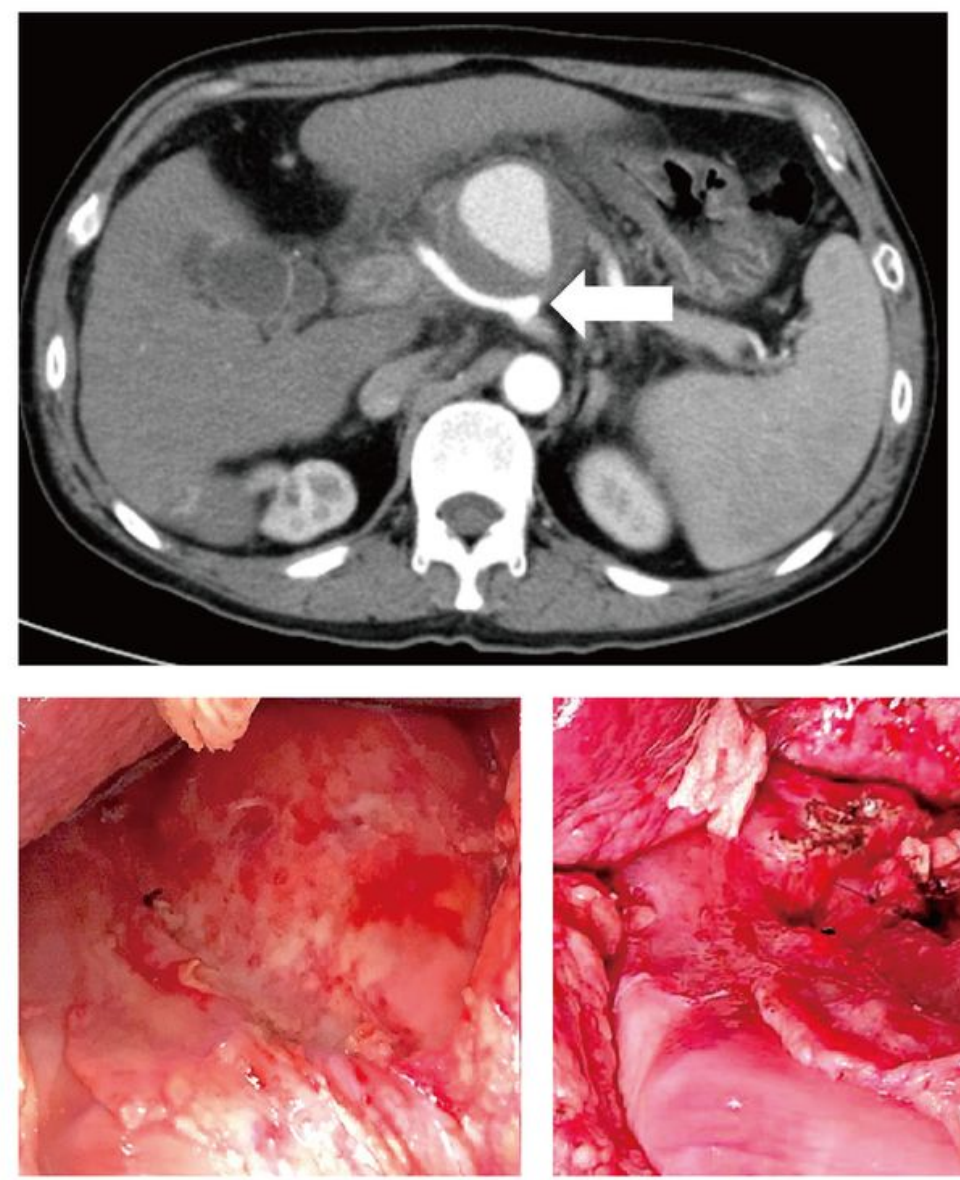

(c)

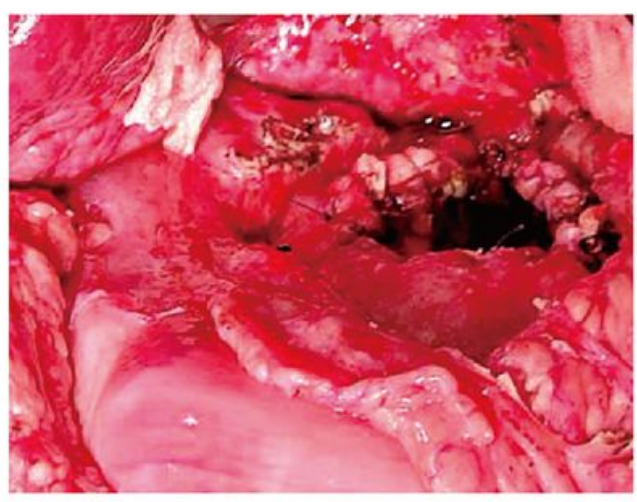

(d) (b)
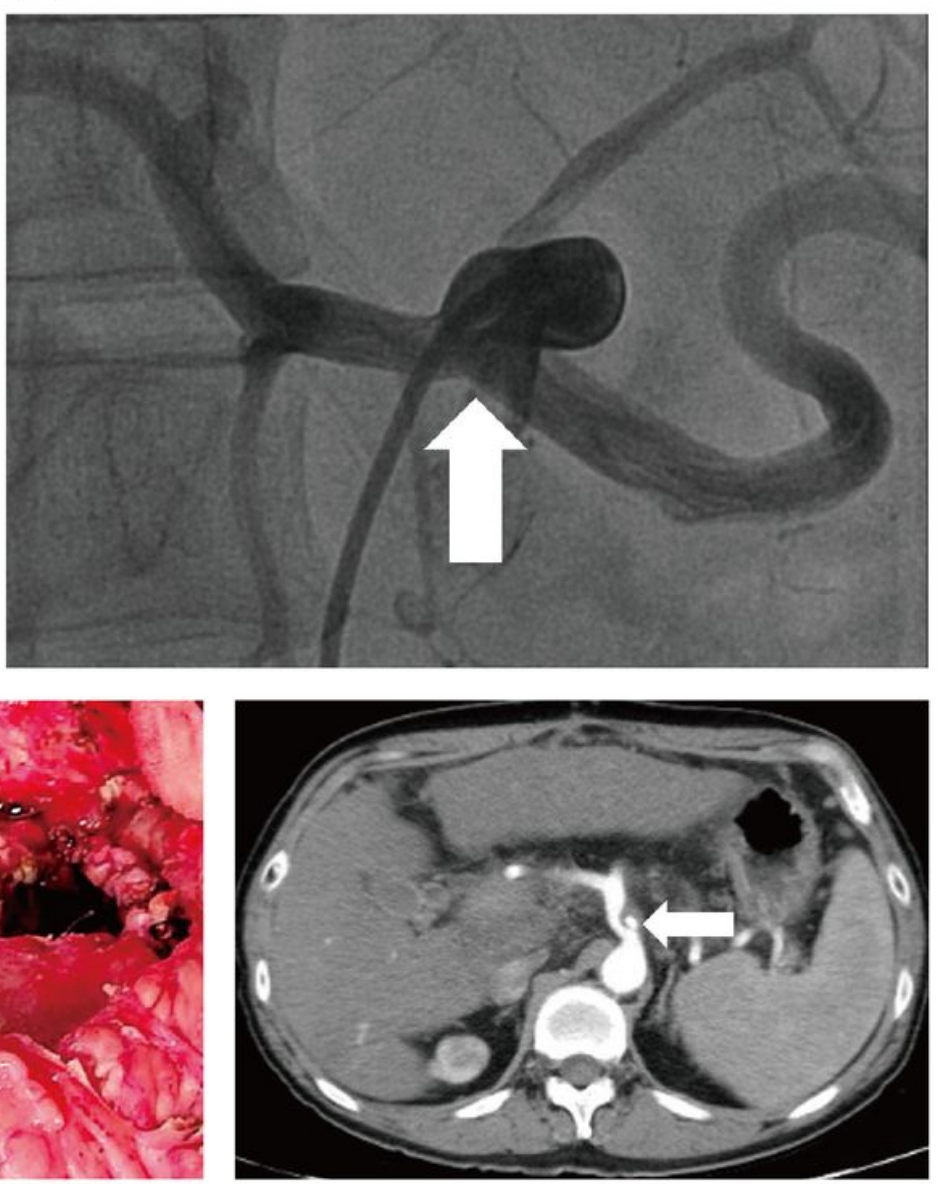

(e)

\section{Figure 1}

(a) CT revealed an aneurysm from the celiac trunk (arrow). (b) The angiography showed a pseudoaneurysm about $6 \mathrm{~cm}$ in diameter arising from the bifurcation of the celiac trunk(arrow). (c) The pseudoaneurysm was about $7 \mathrm{~cm}$ in diameter and challenging to control the feeding vessels from the outside of the aneurysm due to chronic pancreatitis-related dense adhesion. (d) We opened the aneurysm 
directly and directly sutured the bleeder from the inner lumen of the aneurysm. The rim of the pseudoaneurysm cavity was oversewed for hemostasis, and the cavity was packed with an omentum patch to avoid re- accumulated infected fluid. (e) Follow-up CT showed a remaining small pseudoaneurysm $8 \mathrm{~mm}$ in diameter (arrow) six months after the surgery.

(a)
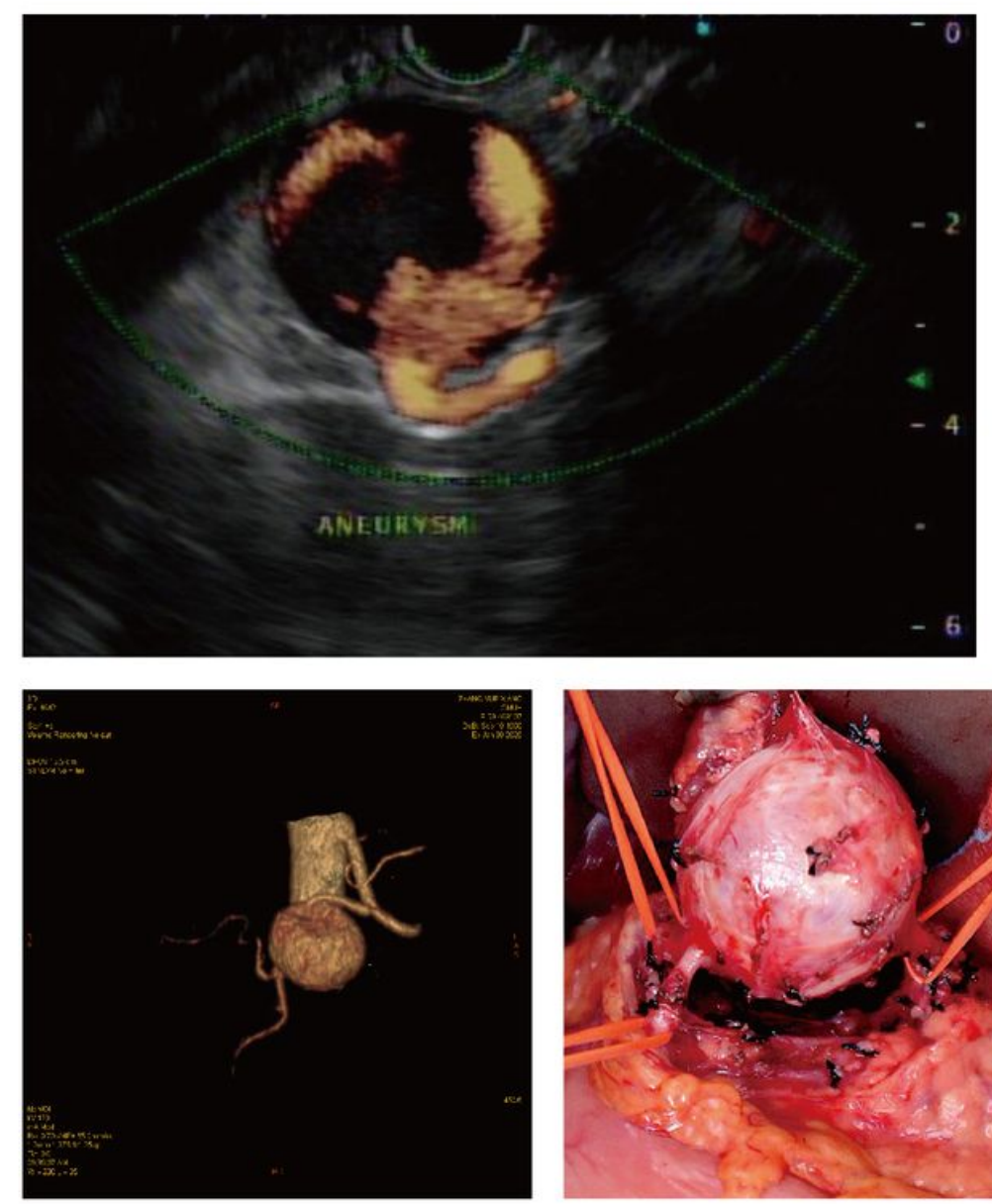

(c)

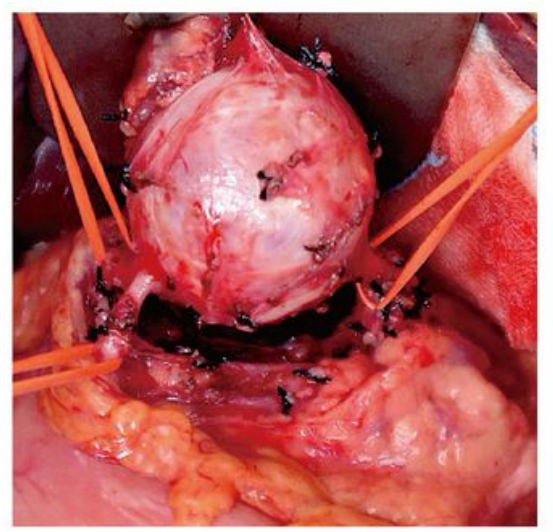

(d) (b)
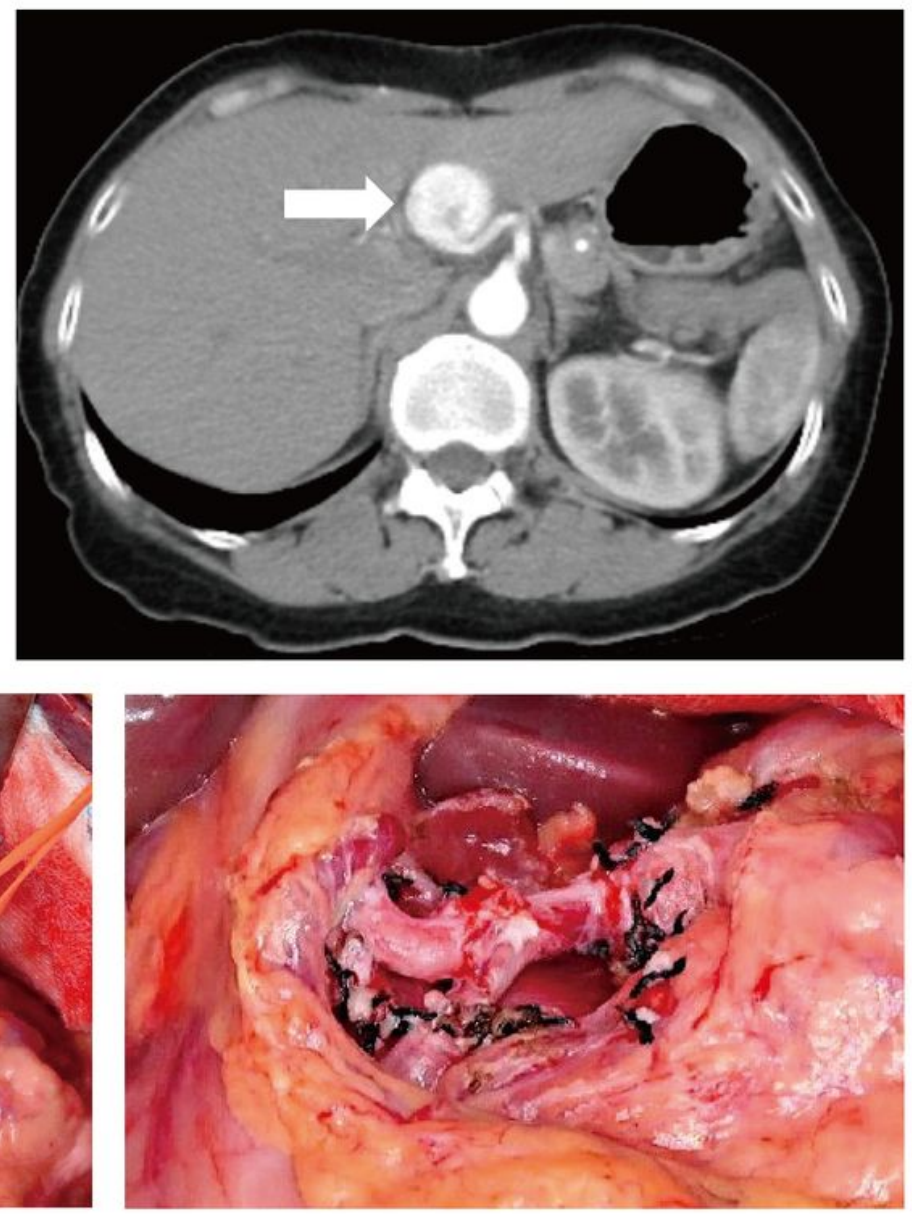

(e)

\section{Figure 2}

(a) Endoscopic ultrasonography showed a common hepatic artery aneurysm (3cm in diameter). (b)(c) The CT scan and CT angiography revealed an aneurysm located between the junction of the common hepatic artery, gastroduodenal artery, and proper hepatic artery. (d) The three feeding vessels of the aneurysm were dissected out and controlled. (e) After excising the aneurysm, we did end-to-end vascular reconstruction between the common hepatic artery and the common orifice of the proper hepatic artery and the gastroduodenal artery. 
(a)

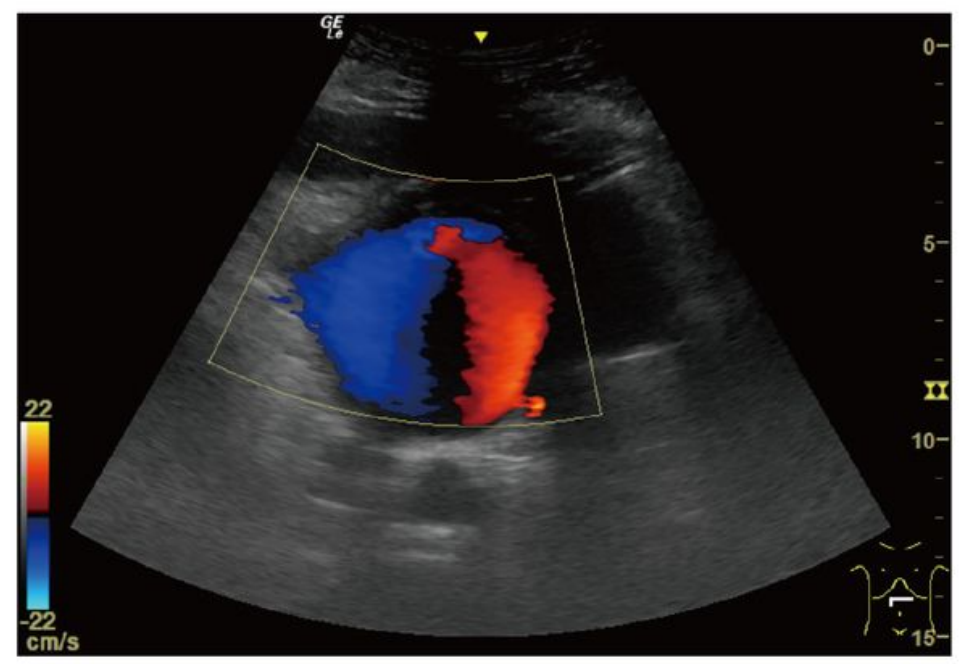

(b)
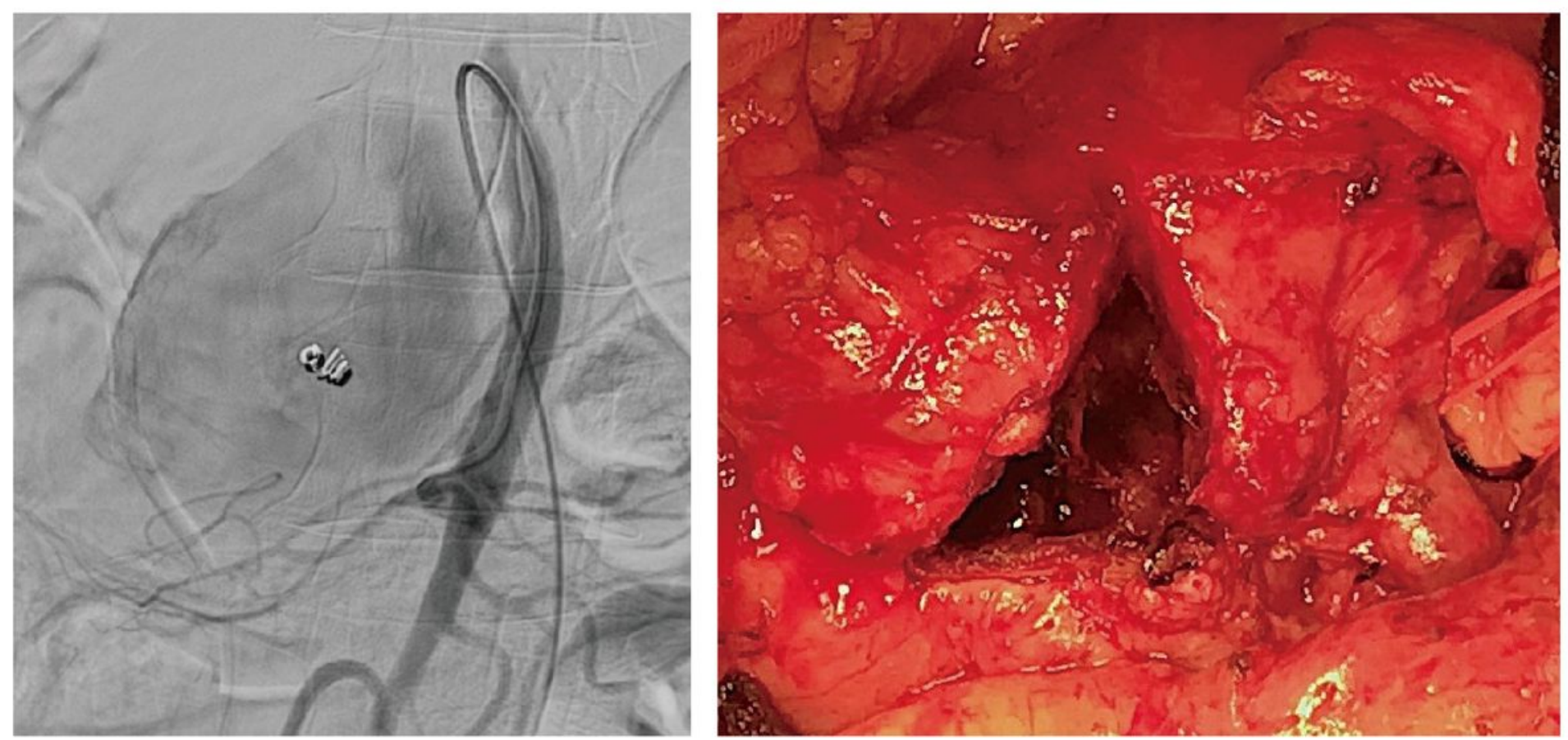

(c)

(d)

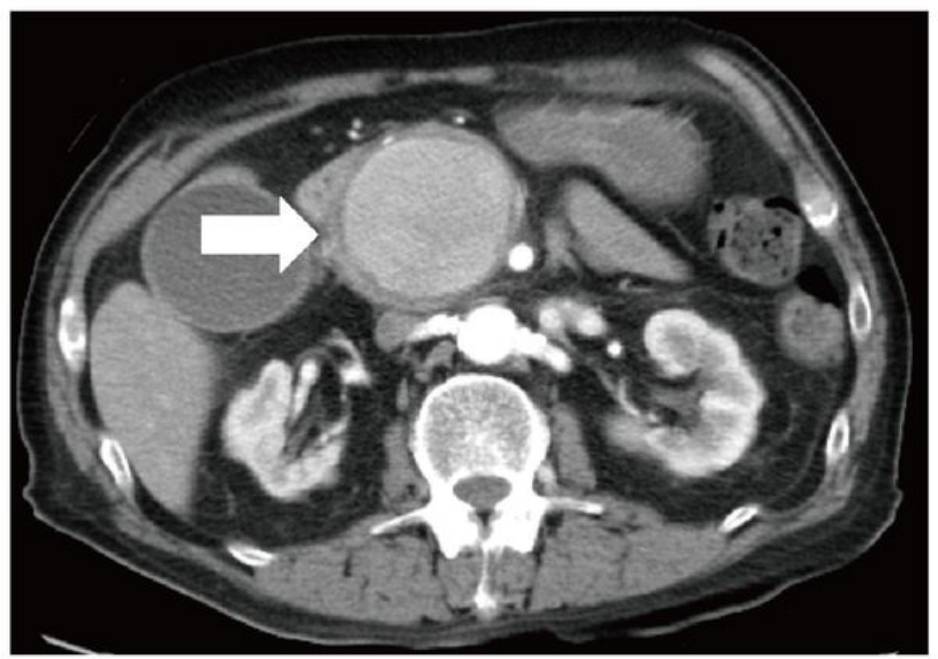

\section{Figure 3}

(a) Abdominal sonography showed a cystic lesion with a "ying-yang" sign that signifies a peri-pancreatic aneurysm. (b) Abdominal CT showed a 6-cm pseudoaneurysm (arrow) arising from the SMA. (c) The angiography showed a SMA aneurysm with two floating micro-coils deployed in a failed coil angioembolization. (d) After ligating the proximal feeding vessel of the aneurysm, we incised the anterior wall of the pseudoaneurysm and sutured the orifice of the distal feeding vessel from the inner lumen of the aneurysm to stop backflow bleeding. 
(a)

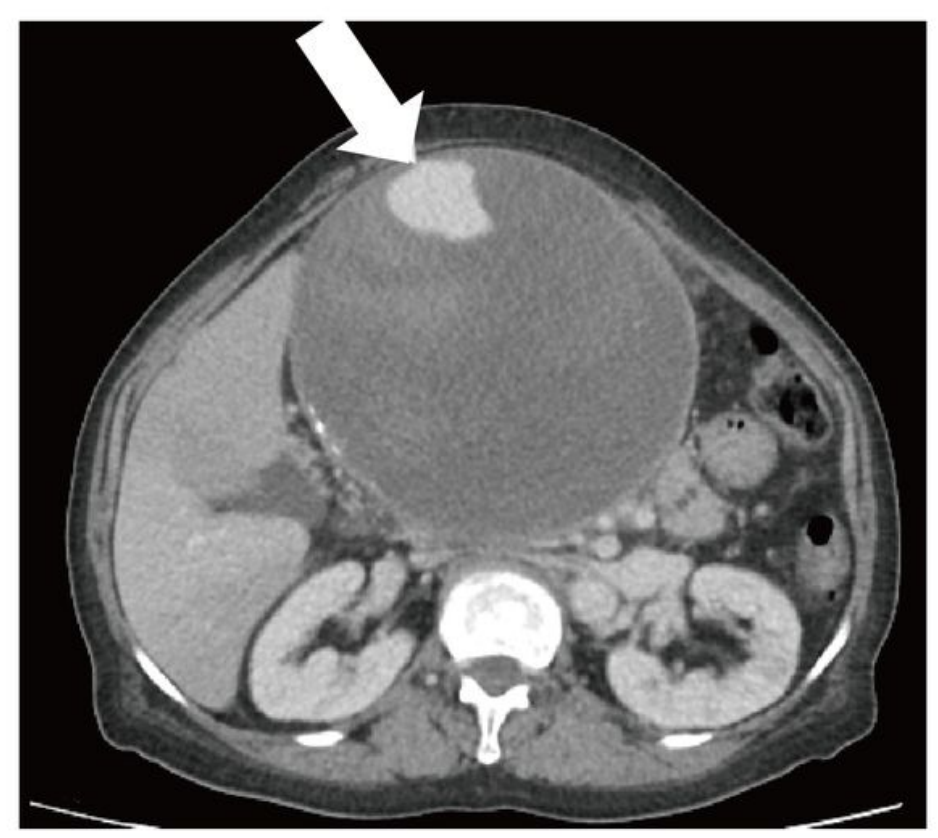

(b)

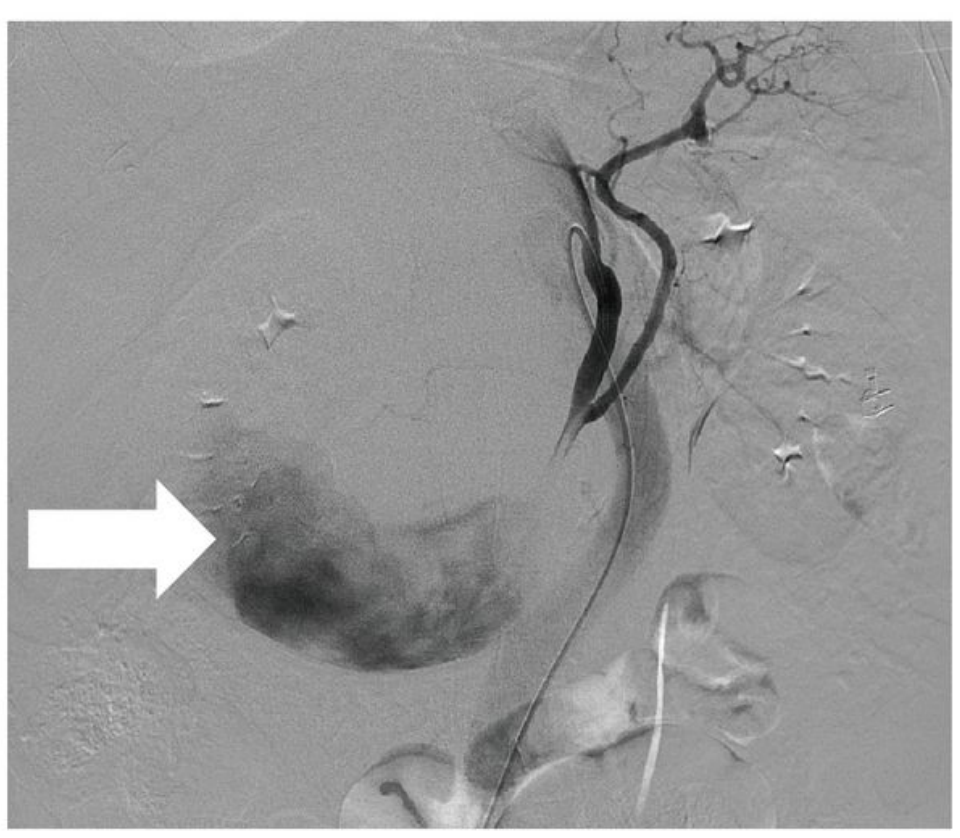

\section{(c)}

\section{Figure 4}

(a) Abdominal CT showed a peripancreatic pseudoaneurysm about $160 \mathrm{~mm}$ (arrow) with mural thrombosis. (b) Angiography confirmed an aneurysm from the dorsal pancreatic artery (arrow), so we performed coil angioembolization via the splenic artery. (c) We offered a salvage operation for partial aneurysmectomy and direct suturing of the orifice of the feeding vessels from the inner lumen of the aneurysm (arrow). 


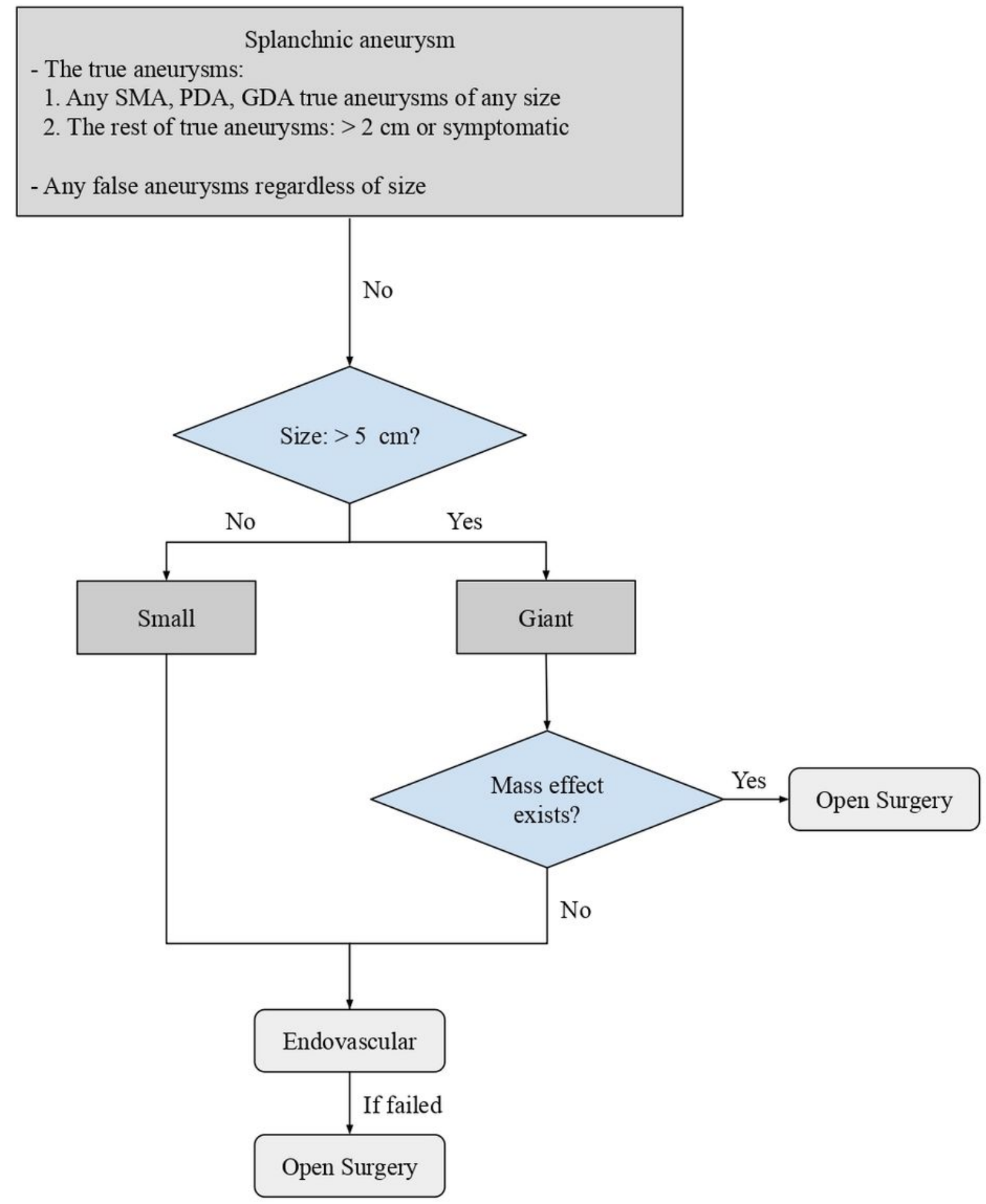

\section{Figure 5}

The decision-making flowchart for splanchnic aneurysms. The management of splanchnic aneurysms in the flowchart is based on their location, size, and clinical situations. 